\title{
MedienPädagogik
}

Zeitschrift für Theorie und Praxis der Medienbildung

\section{Diskurse und Materialität als epistemische Orte der Gewinnung von Orientierungswissen der Medienpädagogik in einer digitalen Welt}

Maik Wunder

\begin{abstract}
Zusammenfassung
Im Beitrag wird eine anthropozentrische Epistemologie zur Gewinnung von Orientierungswissen der Medienpädagogik in einer digitalen Welt problematisiert. Es werden zwei alternative Zugänge, nämlich die diskurstheoretische Perspektive von Michel Foucault und die Perspektive des agentiellen Realismus von Karen Barad, vorgestellt. Hier wird jeweils gefragt, welchen Mehrwert besagte Zugänge zur Generierung von Orientierungswissen in einer digitalen Welt für die Medienpädagogik haben. Hierzu werden für die diskursanalytische Perspektive fünf Thesen zur Diskussion gestellt, welche Überlegungen zur Entschleunigung und Selbstreflexion des Forschungsprozesses sowie der Sichtbarmachung und dem Vergleich von verschiedenen diskursiven Arenen wie auch zur Diskurshaftigkeit von digitalen Daten anstellen. Für die Perspektive des agentiellen Realismus werden vier Thesen entfaltet, die sich mit dem grundlegend sich weitenden Blick durch die materialistische Perspektive und deren Verschränkung mit Diskursen sowie mit einer Erweiterung der Forschungsmethoden befassen und sich schliesslich dem Einbezug der ökologischen Dimension widmen.
\end{abstract}

Discourses and materialism as epistemic realms of the knowledge acquisition of media education in a digital world

\begin{abstract}
In this article, an anthropocentric epistemology for gaining orientational knowledge of media education in a digital world is discussed. Two alternative approaches are presented, namely the discourse theoretical perspective of Michel Foucault and the theory of agential realism by Karen Barad. In each case, the question arises as to what added value these approaches to the generation of orientational knowledge in a digital world might have for media education. For this purpose, for the discourse-analytical perspective five theses are put forward, which reflect on deceleration and self-reflection of the research process, the visualization and comparison of different discursive arenas as well as the discursiveness of digital data. For the perspective of agential realism, four theses are unfolded. They deal with the fundamentally broader view of the materialistic perspective and its entanglement
\end{abstract}


with discourses in general and with an extension of research methods. Finally, they address an inclusion of the ecological dimension.

\section{1. $\quad$ Einleitung}

Fragen wir nach der Orientierung in der digitalen Welt und den Orientierungsleistungen der Medienpädagogik diesbezüglich, dann fällt in einer Art vergröbertem Bild zunächst auf, dass Antagonistinnen und Antagonisten wie Protagonistinnen und Protagonisten gleichermassen zumeist den Menschen als oberste Bezugsgrösse ins Feld führen. So etwa, wenn der Psychiater und Medienkritiker Manfred Spitzer anmerkt: "Schon jetzt ist festzuhalten, dass sich mit der zunehmenden Digitalisierung unseres Lebens Unzufriedenheit, Depressionen und Vereinsamung stark ausbreiten» (Spitzer 2015, 20). Oder aber, wenn der Psychotherapeut Georg Milzner in seinem Sachbuch Digitale Hysterie. Warum Computer Kinder weder dumm noch krank machen positiv konstatiert:

«Meine Grundannahme ist die, dass die Kinder und Jugendlichen, die heute so begeistert Bildschirmspiele spielen, bei Facebook unterwegs sind und ihre medialen Möglichkeiten nutzen, zum Großteil keineswegs degenerieren, sondern sich vielmehr für eine Zukunft rüsten, die weit über den heutigen Stand hinaus von der digitalen Technologie geprägt sein wird» (Milzner 2016, 17).

Für diese binäre Ordnung von Ablehnung und Befürwortung mit dem Gravitationsfeld des Menschen im Mittelpunkt liessen sich noch andere Felder neben dem psychologischen Feld aufführen, von denen aus entsprechend gesprochen wird. Dabei zeigt sich schon an den beiden beispielhaft angeführten Zitaten, dass die Grösse Mensch, auf die sich bezogen wird, keine einheitliche Figur darstellt. Wird der Mensch im Kontext der Digitalisierung auf der einen Seite als pathologisches Objekt aufgerufen, so erscheint er auf der anderen Seite als resilientes und weltoffenes Wesen, das seine Zukunft gestalten wird.

Auf die hier angedeutete Problematik, die unsichere Bezugsgrösse Mensch als Ausgangspunkt und Endpunkt von Orientierungswissen bzw. von Wissen überhaupt zu machen, machte Michel Foucault bereits Ende der 1960er-Jahre aufmerksam. Zu nennen sind hier seine Arbeiten Die Ordnung der Dinge (Foucault 1974), welche den programmatischen Untertitel Eine Archäologie der Humanwissenschaften trägt, und das daran anschliessende Methodenbuch Die Archäologie des Wissens (Foucault 1981). Indem Foucault in der sozialwissenschaftlichen Forschung eine andere Grösse als den Menschen einsetzt, der einen unsicheren Bezugspunkt darstellt, postuliert er, zu einem Mehr an Positivität zu gelangen bzw. als glücklicher Positivist forschend unterwegs zu sein, ohne hierbei einem strengen Positivismus zu verfallen oder im Gegenteil einen harten Strukturalismus zu bemühen (Foucault 1981, 182). Es 
stellt sich dabei die Frage, inwieweit eine solche antihumanistische Perspektive, die Schädelbach in seiner Kritik als «junghegelianische Projektion» (Schädelbach 1989, 240) markiert, für die Medienpädagogik in der digitalen Welt Orientierung bieten kann. Hierzu werde ich Foucaults Anthropologiekritik in den Mittelpunkt stellen, die bisher innerhalb der Sozialwissenschaften kaum beachtet worden ist (Gehring 2015, 190).

Neben Foucault stellen neuerdings unter anderem posthumanistische Entwürfe die epistemische Bezugsgrösse des Menschen infrage (Loh 2018, 11-16). Insbesondere führt dies Karen Barads Entwurf des agentiellen Realismus (Barad 2012) mit der Betonung der Handlungs- und Diskursfähigkeit von Materie vor. Das Besondere an diesem Konzept ist, dass hier Diskurs und Materie miteinander verschränkt werden und sich eine solche Perspektive als Erweiterung der von Foucault vorgetragenen Kritik am Humanismus lesen lässt (Loh 2018, 132).

Nachfolgend werde ich zentrale Aspekte der von Foucault entwickelten Diskurstheorie entfalten, welche den Menschen als epistemische Bezugsgrösse innerhalb der Wissenschaft zu eliminieren sucht. Daran anschliessend werde ich fünf Aspekte benennen, die eine solche Perspektive in Bezug auf Orientierungswissen in der digitalen Welt bzw. der Medienpädagogik beisteuern kann. Analog hierzu verfahre ich mit dem Entwurf von Barad und benenne vier Punkte, die für die Genese von Orientierungswissen in der digitalen Welt bzw. der Medienpädagogik fruchtbar erscheinen.

\section{Diskurse als epistemischer Ort zur Gewinnung von Orientierungswissen in der Medienpädagogik}

\subsection{Die Metapher vom Verschwinden des Menschen und die Humanwissenschaften} In der Ordnung der Dinge formuliert Foucault aus einer Rekonstruktion der Wissensgeschichte - nicht der Wissenschaftsgeschichte (Schneider 2003) - eine Kritik der Humanwissenschaften. Das Buch endet mit der viel diskutierten und beachteten These vom Verschwinden des Menschen (Foucault 1974, 462), die zum zentralen Bestandteil der Kulturwissenschaften geworden ist (Gehring 2015, 190). Foucaults Intention mit diesem Werk ist es, auf der einen Seite mit dem Strukturalismus zu brechen, der die Metaphysik einer totalen Struktur und deren Ausbuchstabierung in der Geschichte postuliert. Auf der anderen Seite will Foucault die Phänomenologie hinter sich lassen, welche in unterschiedlichen Nuancierungen ein transzendentales Subjekt als Ausgangspunkt des Wissens und der Erkenntnis annimmt. So schreibt er in dem Vorwort zur deutschen Ausgabe von Die Ordnung der Dinge: 
«Wenn es aber einen Weg gibt, den ich ablehne, dann ist es der (man könnte ihn, ganz allgemein gesagt, den phänomenologischen Weg nennen), der dem beobachtenden Subjekt absolute Priorität einräumt, der einem Handeln eine grundlegende Rolle zuschreibt, der seinen eigenen Standpunkt an den Ursprung aller Historizität stellt - kurz, der zu einem transzendentalen Bewußtsein führt» (Foucault 1974, 15).

Foucault lässt sich hierbei von den Ausführungen von Gaston Bachelard leiten, der insbesondere, inspiriert durch die Erkenntnisse der modernen Physik zu Beginn des 20. Jahrhunderts, einen radikalen Bruch zwischen der sinnlichen und der wissenschaftlichen Erkenntnis propagierte (Bachelard 1987, 345). «Die Welt, in der man denkt, ist nicht die Welt, in der man lebt» (Bachelard 1978, 129). Das Element, auf das Bachelard hier verweist, ist das des epistemologischen Bruchs, der bewirkt, «dass die Dinge plötzlich nicht mehr auf die gleiche Weise perzipiert, beschrieben, genannt, charakterisiert, klassifiziert und gelernt werden» (Foucault 1974, 269) können. Die Geschichte des Wissens ist damit durch Diskontinuitäten und Uneinheitlichkeit gekennzeichnet, stellt also keineswegs den sukzessiven Aufstieg und Fortschritt einer immer mehr zu sich selbst kommenden Vernunft dar. Vor diesem Hintergrund rekonstruiert Foucault drei durch epistemologische Brüche zu unterscheidende Epochen innerhalb der Wissensgeschichte. Diese Epochen sind durch jeweils unterschiedliche Wissensordnungen bzw. Episteme gekennzeichnet, welche die Bedingung für Wissen und dessen Anordnung überhaupt darstellen (Foucault 1974, 24-25). Es handelt sich also bei den Epistemen um kognitive Ordnungsschemata, spezifische Logiken der Erkenntnis, die auf der Oberfläche der Wissensobjekte zunächst unsichtbar sind (FinkEitel 1997, 38; Sarasin 2006, 71).

Im Folgenden skizziere ich knapp die ersten beiden Epochen, um vor diesem Hintergrund (a) deutlicher das Problem darlegen zu können, auf welches Foucaults Arbeit aufmerksam macht, und (b) um Foucaults Lösung als Konsequenz dieser Befunde plausibel zu machen.

Für die Jahre 1500 bis 1650 konstatiert Foucault das Epistem der Ähnlichkeit: «Den Sinn zu suchen, heisst an den Tag zu bringen, was sich ähnelt. Das Gesetz der Zeichen zu suchen, heisst die Dinge zu entdecken, die ähnlich sind» (Foucault 1974, 60). Daher sind Sprache und Dinge zwei unterschiedliche Seiten einer Medaille, einzig und allein durch den Sündenfall gab es einen kleinen Bruch zwischen beiden Ebenen, was die Notwendigkeit des Kommentars evozierte (Frietsch 2014, 45). Der Mensch erscheint in dieser Wissensordnung zwar als Individuum, aber nur in Abhängigkeit von einem allmächtigen Gott bzw. der Natur. Dieser starke Gottesbezug lässt dann auch die Fragen nach der Erkenntnisfähigkeit des Menschen in den Hintergrund treten (Sarasin 2006, 84-85). Foucault macht deutlich, wie fremd uns heute ein Denken in Ähnlichkeiten ist, indem er darauf hinweist, dass mit Bacon und Descartes die 
Ähnlichkeit nur eine unsichere, provisorische Stellung einnimmt, die mitunter an der Grenze zur Täuschung liegt (Foucault 2001b, 622). Das Zeitalter der Ähnlichkeit wird vom Zeitalter der Repräsentation bzw. der Klassik abgelöst, einer Zeit, die Foucault zwischen 1650 und 1800 ausmacht. Die Ähnlichkeit, die zwischen Dingen und Zeichen vermittelt, verschwindet hier und an deren Stelle wird die Repräsentation gesetzt. Die Bindung der Zeichen an das Innere der Dinge verschwindet und wird durch die Repräsentation der Dinge in den Zeichen ersetzt. Die Sprache, so die Vorstellung, ist im Hinblick auf die Ordnung der Dinge vollkommen transparent, sie verkörpert eine Ordnung aus Begriffen, Differenzen und Identitäten, bleibt aber an die Dinge rückgebunden und spiegelt diese massstabsgetreu wider, in sich selbst setzt sie ihr eigenes Ordnungsschema frei, bedarf aber keiner Interpretation mehr (Fink-Eitel 1997, 40). Allerdings kommt der Mensch innerhalb dieses Repräsentationssystems nicht vor: «Aber es gab kein erkenntnistheoretisches Bewusstsein vom Menschen als solchem. Die klassische episteme gliedert sich nach Linien, die in keiner Weise ein spezifisches und eigenes Gebiet des Menschen isolieren.» (Foucault 1974, 373) Daher kann das klassische Zeitalter auch als ein «subjektloses Denken» (Schneider 2004, 70) angesehen werden, in dem ein selbstregelndes Zeichensystem die Abbildungen und Vorstellungen indiziert. Die Vorstellung ist damit die Vorstellung der Repräsentation in ihrer Funktion (Dahlmanns 2008, 28).

Ein fundamentaler Wandel in der Wissensordnung setzt dann im 19. Jahrhundert ein, das Foucault als das Zeitalter des Menschen markiert, eine Epoche, die bis heute andauert und deren Analyse die Grundlage für die Kritik der Humanwissenschaften bildet. Auf Basis der Episteme der vorangegangenen Ordnung wird deutlich, dass der Mensch vor dem Ende des 18. Jahrhunderts nicht existierte, vielmehr, so Foucault, ist er eine «völlig junge Kreatur, die die Demiurgie des Wissens eigenhändig vor noch nicht einmal zweihundert Jahren geschaffen hat.» (Foucault 1974, 373) Im Rahmen von Kants drei Kritiken wird deutlich, dass der Mensch nunmehr versucht, sich selbst als das repräsentierende Subjekt, als den Grund der Repräsentation zu repräsentieren (Wunder 2018a, 34). Die Tableaus und Ordnungsschemata der klassischen Epoche waren nicht mehr in der Lage, eine sichere Erkenntnis zu liefern und die Einheit der Dinge sicherzustellen. Damit eröffneten sich im Grunde nur zwei Wege, um mit dem Ende der Repräsentation umzugehen. Auf der einen Seite werden ein transzendentales Ich bzw. apriorische Strukturen der reinen Vernunft als Ausgangspunkt wissenschaftlichen Suchens eingesetzt (Sarasin 2006, 80). Damit stellt der Mensch zum einen eine empirische Realität dar, die erforschbar ist, und ist gleichermassen die Bedingung der Möglichkeit von wissenschaftlicher Erkenntnis (Schneider 2004, 77). Dieser Akt lässt sich nur durch eine Verfinsterung der Welt ermöglichen, die eben durch das Bewusstsein nicht erreicht werden kann, da von diesem transzendentalen Bewusstsein alle formellen Bedingungen des Empirischen abhängen und es sich im Grunde nur selbst widerspiegelt, nicht aber die Dinge an sich (Fink-Eitel 1997, 42). 
Auf der anderen Seite werden die Bedingungen der Erfahrung auf das Objekt transferiert, die empirischen Wissenschaften gewinnen damit an Bedeutung - auch in der Beschreibung des Menschen als Objekt. Dieser lässt sich nunmehr durch Leben, Arbeit und Sprache als empirisch erforschbare Bereiche der Wirklichkeit bestimmen (Dahlmanns 2008, 30-31). Der Mensch, so Foucault, erscheint also als «empirischtranszendentale Dublette, weil er ein solches Wesen ist, in dem man Kenntnis von dem nimmt, was jede Erkenntnis möglich macht» (Foucault 1974, 384). Neben dieser Dublette, «das Wesen Mensch», erscheinen zwei weitere Dubletten in der modernen Wissensordnung, die von Foucault kritisiert werden. Zum einen die Dublette des Cogito. Der moderne Mensch realisiert, dass er in einer Sprache spricht, die er nicht selbst hervorgebracht hat, dass er einen Körper hat, der sein Leben erhält, der sich aber in seinen Vitalfunktionen weitestgehend seinem direkten Zugriff entzieht, und dass er zutiefst durch die Mechanismen des Produktions- und Reproduktionssystems konstituiert wird. Diese Grössen entziehen sich dem Cogito. Es wird vom Ungedachten her bestimmt, eine vollständige Einholung dieses Ungedachten etwa durch die Psychoanalyse scheint nicht möglich, da das Ungedachte/Unbewusste ein konstitutives Element für das Ego darstellt. Es führt also kein direkter Weg in der modernen Wissensordnung vom «Ich denke» zum «Ich bin», weil sich dem Denken immer etwas entzieht (Dahlmanns 2008, 35-36). Die andere Dublette hängt eng mit dieser hier geschilderten zusammen. Hier wird nach dem Ursprung des Menschen jenseits von Leben, Arbeit und Sprache in einer mystischen Vergangenheit gesucht. Das Dasein kann nur in einem existenziellen Akt der Verzweiflung den Verlust des Ursprungs auf sich nehmen, um diesen dann als Abwesenheit sich zu vergegenwärtigen (Heidegger 2010, 29; Nietzsche 1994, 16). Auf der anderen Seite kann etwa im Denken von Hegel und Marx der Ursprung in einer Art Wiederkehr zu sich selbst in Zukunft zurückkehren und die Entfremdungen der obigen Trias auflösen, indem sich das Denken selbst auflöst (Dahlmanns 2008, 38).

«Und dadurch, in dieser unendlichen Aufgabe, den Ursprung möglichst nahe und möglichst fern von sich zu denken, entdeckt das Denken, daß der Mensch nicht mit dem zeitgenössisch ist, was inn existieren läßt - oder mit dem, von wo ausgehend er ist; sondern, daß er in einer Kraft gefangen ist, die ihn verstreut, ihn fern von seinem eigenen Ursprung hält, aber ihm seinen Ursprung in einem unmittelbaren Bevorstehen verheißt, das vielleicht für immer ihm entzogen bleibt» (Foucault 1974, 403).

Das Paradoxe dabei ist, dass Arbeit, Leben und Sprache Leistungen des Menschen sind, diese ihn aber zugleich konstituieren und damit überholen (Fink-Eitel 1997, 43-44). «Man kann zu ihm nur Zugang durch seine Wörter, seinen Organismus, die von ihm hergestellten Gegenstände haben» (Foucault 1974, 378-379). Der Mensch erscheint als «unterworfener Souverän» (Foucault 1974, 379). Seine Endlichkeit ist in 
Arbeit, Leben und Sprache verkörpert, die auf der einen Seite als positive Bedingung von Erkenntnis fungieren, andererseits die Fragilität des Menschen als Bezugspunkt, insbesondere der Humanwissenschaften, offenbaren. Hier setzt Foucaults Kritik der Humanwissenschaften ein. Denn nicht der Mensch hat sich selbst als Subjekt und Objekt konstituiert, sondern dieser Vorgang ist ihm entzogen und unterliegt den epistemischen Konfigurationen (Dahlmanns 2008, 39). Dies ist der eine Kritikpunkt Foucaults an den Science humaines, die sich besser mit Menschenwissenschaften übersetzen lassen und alle Sozialwissenschaften umfassen, die sich in eine hermeneutische Tradition stellen (Gehring 2015, 193). Indem diese Wissenschaften auf Basis des «Primat[s] der Repräsentation» (Foucault 1974, 435) arbeiten, sind sie instabil, da die Repräsentation nunmehr selbst ein Teil der empirischen Ordnung sein muss und analysiert werden kann. Derartige Unternehmungen werden somit zirkulär, instabil und können kein Allgemeines erreichen (Dahlmanns 2008, 43). «Sie gehen von dem, was der Repräsentation gegeben wird, zu dem, was die Repräsentation möglich macht, was aber wiederum eine Repräsentation ist» (Foucault 1974, 436). Da die jungen Humanwissenschaften auf keine vorherige Episteme zurückgreifen können, werden die klassischen Wissenschaften, die sich mit dem Leben, der Sprache und der Arbeit befassen, für diese konstitutiv und markieren gleichzeitig ihre Grenze (Dahlmanns 2008, 40-42). Da aber diese Wissenschaften als Quasi-Transzendentalien (Foucault 1974, 307) für die Repräsentation unbewusst sind, die Humanwissenschaft sich aber ihrer bedient, kann niemals so etwas wie ein Wesen des Menschen zum Vorschein kommen, da eben der Mensch durch diese Elemente erst ins Leben gerufen wird (Foucault 2001c, 841). Zum anderen sind insbesondere die Humanwissenschaften in das Gefüge der Macht eingebunden, welche es erlaubt, den Menschen biopolitisch zu formen (Gehring 2015, 195-197).

Vor diesem Hintergrund wird Foucaults Metapher vom Ende des Menschen dekodierbar. Verschwinden wird nicht der einzelne Mensch als wortbegabtes Wesen unter anderen Wesen, sondern der Mensch als empirische Grösse der einzelnen Wissenschaften und als Grund der modernen Wissenschaften (Gehring 2015, 191).

«Wenn ich sage, der Mensch habe aufgehört zu existieren, will ich natürlich nicht sagen, der Mensch als biologisches oder soziales Wesen sei von unserem Planeten verschwunden. Das soziale Funktionieren ist natürlich das Funktionieren der Individuen in ihrer wechselseitigen Beziehung» (Foucault 2001d, 790).

So wird der Mensch dezentriert, je mehr z.B. deutlich wird, dass etwa die Sprache den Menschen fundamental konstituiert: 
«In dem Erdbeben, das uns heute erschüttert, müssen wir vielleicht die Geburt einer Welt erblicken, in der man wissen wird, dass das Subjekt nicht eins ist, sondern zerrissen; nicht souverän, sondern abhängig; nicht absoluter Ursprung, sondern stets wandelbare Funktion» (Foucault 2001a, 1002-1003).

Indem insbesondere die analytische Philosophie mit den Arbeiten Wittgensteins (Wittgenstein 2000) eine sprachkritische Wende bzw. einen linguistic turn (Rorty 1975) vollzieht, gewinnt die Sprache immer mehr an Bedeutung, stellt im Grunde den Wirklichkeitsbezug schlechthin dar (Landwehr und Stockhorst 2008, 76) und verweist den Menschen auf eine «heitere Inexistenz» (Foucault 1974, 461) zurück. Unter diesem Eindruck sucht Foucault nun seinen eigenen Weg für die Erforschung der sozialen Welt, eben ohne ein wissendes Subjekt vorauszusetzen, indem er eine eigene Form der Diskursanalyse entwickelt.

\subsection{Diskursanalyse als Lösung der aufgezeigten Probleme}

Foucault versteht unter dem Diskurs eine systematisch-regelhafte Abfolge von Aussagen, die eine spezifische Funktion haben und unter spezifischen Bedingungen erscheinen oder eben nicht erscheinen. Aussagen konstituieren den Referenten, die Subjektpositionen, die Aussagefelder und die materielle Existenz und sind mit einem Formationssystem verwoben, das äquivalent zu den Aussagefunktionen Gegenstände, Äusserungsmodalitäten, Begriffe und Strategien regelhaft anordnet. Dieses regelhafte System kann durch die Erfassung der verstreut liegenden Aussagen rekonstruiert werden, um anschliessend in Form eines abduktiven Schliessens (Peirce 1991, 129) auf eine Wissensordnung, sprich eine Sozio-Episteme, zu rekurrieren, welche den Diskurs trägt. Daher muss die foucaultsche Diskursanalyse als ein post-strukturales Unterfangen verstanden werden, das mit der Metaphysik der Struktur bricht, sie für die Geschichte und Veränderlichkeit öffnet und dennoch an die Existenz einer Strukturalität glaubt, die nicht jenseits des Gesprochenen ist, sondern deren positive Ermöglichung oder Begrenzung darstellt (Diaz-Bone 2004, 52).

Einzuwenden ist allerdings, dass Diskursanalysen, insbesondere wenn sie sich auf die Gegenwart beziehen, aus drei Gründen immer unvollständig sein müssen. Erstens bleiben diese auf Text beschränkt, sie sind nicht in der Lage, etwa technische Artefakte in ihrem Akteursstatus zu erfassen (Latour 2010). Dies mag zweitens daran liegen, dass die Diskursanalyse grundlegend von einer Trennung zwischen Natur und Kultur/Gesellschaft ausgeht und daher ein intraaktiv verschränktes Werden von Materialität und Diskurs nicht erfassen kann (Barad 2012). Drittens erscheint es unmöglich, die eigene Episteme, in der man steht, vollständig zu erfassen, da die Wissensordnung, mit der man die Rekonstruktion durchführt, selbst Teil dessen ist, was man untersucht (Foucault 1981, 188). Schliesslich ist es nicht möglich, alle Elemente des Diskurses zu erfassen. 
Dieses Unvollständigkeitsproblem mag mit Sicherheit ein Grund dafür sein, warum die foucaultsche Diskurstheorie nur marginal in der Erziehungswissenschaft (Ricken 2007; Balzer 2004) und so gut wie gar nicht in der Medienpädagogik (Dander 2017, 51) ihre Berücksichtigung findet - obwohl Foucaults Arbeiten mittlerweile zu einem Klassiker der Sozialwissenschaft geworden sind (Angermüller 2004) und die von ihm rekonstruierten neoliberalen Sprachspiele als weitestgehend salonfähig erscheinen (Witte 2005, 326). Der andere Grund mag daher mit eben dieser Kritik und der damit verbundenen Dezentrierung und Entthronisation eines wissenden und handelnden Subjekts durch die foucaultsche Perspektive zu tun haben. Denn eine solche Sichtweise irritiert den Kern eines spezifischen erziehungswissenschaftlichen Selbstverständnisses und deren Legitimation sowie die nicht aufzulösende Verschränkung von Theorie und Praxis, die sich nicht in einer reinen Beschreibung und Reflexion erschöpfen kann (Ricken 2007, 158). Nicole Balzer wirbt für ein nichtoppositionelles Denken in der Erziehungswissenschaft beim Umgang mit Foucault, das sich weder auf die Seite der Freiheit bzw. der Macht noch auf die Seite der Autonomie bzw. der Fremdbestimmung stellt (Balzer 2004, 16). Wird diese oppositionelle Struktur verlassen, kann durchaus mit der angezeigten Perspektive ein Orientierungswissen in der Medienpädagogik gewonnen werden. Dieses Orientierungswissen vermeidet dann im Rahmen von Diskursanalysen die Implikationen einer kritischen Erziehungswissenschaft, welche droht, traditionelle pädagogische Vorstellungen zu bemühen, dass etwa Ökonomie und Bildung sich wechselseitig ausschliessende Elemente seien oder dass Fremdbestimmung und Selbstbestimmung zwei sich widersprechende Perspektiven darstellten (Ricken 2007, 162). Daher möchte ich zum Abschluss fünf Punkte zur Diskussion stellen, welchen Orientierungsgewinn die Medienpädagogik aus der Analyse von Diskursen haben kann. Der erste Punkt bezieht sich auf die Besonderheit von diskursanalytischer Forschung, die mit grossen Datenmengen über einen längeren Zeitraum arbeitet. Dies dokumentieren etwa Foucaults Arbeiten zur Ordnung der Dinge (Foucault 1974) oder auch die Studien zur Geburt der Klinik (Foucault 2002).

Der zweite Punkt greift die diskursanalytische Frage nach der epistemologischen Situierung der Forschenden im Feld auf. Dies entbindet von transzendentalen Setzungen zum einen aufseiten des forschenden Subjekts, da es Teil der diskursiven Hervorbringungsmechanismen ist, zum anderen aufseiten des Forschungsdesigns, das sich entgegen den humanistisch-hermeneutischen Traditionen nur auf das Immanente bezieht, ohne nach Sinngehalten in einem Hintergrund zu suchen. Im dritten und vierten Punkt wird der Aspekt thematisiert, dass Diskurse Macht haben, über die Köpfe von menschlichen Akteuren hinweg Wirklichkeit zu konstituieren. Und schliesslich werden Überlegungen angestellt, ob nicht das Digitale als diskursives Element gefasst werden kann und daher genau wie jedes andere semiotische Material zu behandeln ist. 


\subsection{Diskursanalyse als Orientierungsinstrument der Medienpädagogik in einer} digitalen Welt

(a) Entschleunigung. Diskursanalyse kann durch ihr langwieriges und durch grosse Datenmengen gekennzeichnetes Forschungsdesign zur Entschleunigung von medienpädagogischen Performanzen beitragen und es so erleichtern, von den aus dem aktuellen Tagesgeschäft herangetragenen Forderungen zurückzutreten. Das stark reflexive Element und das des epistemologischen Bruchs verhindert, dass die Medienpädagogik als Gehilfin von stark beschleunigten technologischen Innovationen adressiert wird, die massiv das Bildungssystem zu okkupieren versuchen.

(b) Selbstreflexion. Diskursanalyse dient der Selbstreflexion in Bezug auf die eigenen pädagogischen Performanzen und Paradigmen der Forschung. Sie kann zum einen die eigene Situierung in ein sozio-epistemisches Feld kenntlich machen. So zeigt etwa eine diskursanalytische Arbeit, dass innerhalb der medienpädagogischen Spezialdiskursarena mit neoliberalen Figuren operiert wird, indem etwa die digitalen Artefakte als Instrumente konstruiert werden, die ein individualisiertes, selbstgesteuertes Lernen möglich machen und hierbei mit den Subjektkonstruktionen der Lernenden hochgradig figuriert sind. Diese werden als individualisiert Lernende adressiert, deren individualisierter Lernprozess recht häufig mit ökonomischen Begriffen wie Effizienz oder Produktivität gekoppelt werden (Wunder 2018a, 108-114).

Zum anderen kann Diskursanalyse als reflexives Instrument betrachtet werden, um den eigenen Forschungsprozess hinsichtlich der epistemologischen Paradigmen zu hinterfragen. Sie regt dazu an, Theorie, Methodologie und Methoden in ein kohärentes Passungsverhältnis zu bringen (Diaz-Bone und Widmer 2018, 150), ohne eben hierbei mit transzendentalen Entitäten als konstitutiven Elementen arbeiten zu müssen. Vielmehr wird auf der Ebene des Diskurses verblieben, also in einem immanenten Raum des tatsächlich Gesagten. Hierin sehe ich im Grunde den grössten Orientierungsgehalt, den eine solche Analyse aufgrund der von Foucault problematisierten fragilen Bezugnahme der Humanwissenschaften liefern kann, da sie eben mit positiven Gegenständen arbeitet und damit in ihren Konstruktionen - folgt man Foucault - zu einer grösseren Stabilität, Allgemeinheit und Validität gelangt.

(c) Diskurse und Diskursarenen sichtbar machen. Diskursanalyse kann Orientierung in der digitalen Welt bieten, indem durch diese sichtbar gemacht werden kann, welche Diskurse und welche Diskursarenen aktuell bestimmend sind. Sie kann zudem identifizieren, dass antagonistische Diskurse durchaus in einer gleichen SozioEpisteme wurzeln. So konnte gezeigt werden, dass Ablehnung und Befürwortung 
von digitaler Bildung beide gleichermassen in einer Sozio-Episteme der Beschleunigung situiert sind (Wunder 2018a, 182ff.). Hieraus resultiert der Orientierungswert, dass man durch eine solche gewonnene Metaperspektive einen breiteren diskursiven Handlungsraum eröffnen kann, der jenseits einer Dialektik von These und Antithese liegt. Dieser Raum ermöglicht dem medienpädagogischen Feld, von dem aus gesprochen wird, sich umfassender zu Diskursen und in Diskursen zu positionieren und mit diesen zu operieren. Diskurse sind nämlich - dies macht Foucault deutlich - von innen heraus in sich selbst mächtig und sind Teil der Macht, die von aussen auf den Diskurs einwirkt und diesen dazu benutzt, Kontrolle auszuüben (Foucault 2000, 17). Daher geht Diskursanalyse stets mit Machtkritik einher.

(d) Zusammenhänge zwischen einzelnen diskursiven Arenen herstellen. Diskursanalyse kann fernerhin zur Orientierung in der digitalen Welt beitragen, da sich mit dieser identifizieren lässt, wie einzelne diskursive Arenen miteinander zusammenhängen, in denen Wissen zirkuliert, produziert und reproduziert wird. So können hier regelhafte Wiederholungen und Iterationen nachgezeichnet werden und es kann geprüft werden, welcher Ort, vom dem aus gesprochen wird, sich gerade als besonders innovativ oder stabilisierend erweist. So konnte etwa in meiner Untersuchung (Wunder 2018a) gezeigt werden, dass es im Rahmen einer situativen Diskursarena zur digitalen Bildung gegenüber höherdimensionalen Diskursarenen wie der Spezial- oder Interdiskursarena zu Iterationen in Bezug auf die Rolle der Lehrkräfte gekommen ist. Diese werden hier als starke Akteure und zentrale Instanzen konstruiert, die den Prozess der Digitalisierung von Schule tragen und forcieren, während diese Akteure in den anderen beiden Diskursarenen eher als marginale und hemmende Grösse aufgerufen werden (ebd., 180).

(e) Digitale Zeichen als Diskurselemente. Digitale Zeichen, wie sie etwa im Rahmen von Big Data oder Algorithmen produziert werden, lassen sich wie in post-strukturalistischen Modellen als Trennung zwischen Signifikat und Signifikant fassen und damit als Diskursmaterial, das keineswegs auf eine Wirklichkeit jenseits ihrer selbst verweist, sondern auf die Wirklichkeit der Totalität der Zeichen, die regelhaft bzw. musterhaft sich stets neu (re)-kombinieren (Nassehi 2019, 102ff.) und damit Begriffe, Subjektpositionen, Gegenstände und Strategien hervorbringen, von denen sie sprechen (Foucault 1981, 74). Die digitalen Daten gleichen damit analogen Aussagen im foucaultschen Sinn, deren Existenzbedingungen sich rekonstruieren lassen (Gnosa 2019, 58). Hierzu bedarf es grundlegender Kenntnisse ihrer Operationsweisen, um die entsprechenden darin eingeschriebenen Machtstrukturen zu identifizieren. Die Macht von algorithmisiertem Diskursmaterial «liegt in den nicht sichtbaren Prozessen, die prägen, was als Wissen gilt, und diese Wissensbestände strukturieren» (Bächle 2016, 47). 
Zugegebenermassen, entsprechende Punkte liessen sich auch im Rahmen einer kritisch operierenden Medienpädagogik oder in phänomenologisch-hermeneutischen Traditionen postulieren. Allerdings wird mit der posthumanistischen Perspektive, die den Menschen dezentriert und damit den Fokus auf Elemente richtet, eine andere Epistemologie evoziert, die wie erwähnt den Anspruch erhebt, ein Mehr an Positivität im Rahmen der Forschung zu verwirklichen.

«Der Positivismus der Archäologie bedeutet nun keine naive Deskription des Vorgefundenen, sondern beinhaltet die Unterstellung, dass sich in den diskursiven Formationen die Regeln finden lassen, die das jeweils historisch-spezifische a priori des Denkens im Diskursfeld selbst sind. Das transzendentale Modell ist somit nun das positiv gegebene Spiel der Regeln, die der diskursiven Praxis inhärent sind und die gleichzeitig (als unterstellte) Bedingung der Analyse und Objekt der Analyse sind» (Diaz-Bone 2010, 184).

\section{Materialität als epistemischer Ort zur Gewinnung von Orientierungswissen in der Medienpädagogik}

\subsection{Das Digitale als Infragestellung des Menschen}

Der letzte der fünf oben genannten Punkte verweist auf eine vierte Grösse, die neben der von Foucault offerierten Trias aus Leben, Arbeit und Sprache, die zur Infragestellung des Menschen führen, im 21. Jahrhundert an Kontur gewinnt und den beschriebenen Prozess weiter vorantreibt (Bostrom 2018): «Ein Gespenst geht um in der globalisierten Gesellschaft - das Gespenst der Digitalisierung» (Precht 2018, 15). Hierbei darf nicht neben der skizzierten Diskursivität des Digitalen übersehen werden, dass dieses eine konkrete materielle Basis besitzt.

"Schriften und Texte (unter Einschluss des Textes, den ich eben vorlese) existieren mithin nicht mehr in wahrnehmbaren Zeiten und Räumen, sondern in den Transistorzellen von Computern» (Kittler 1993, 225).

Im Entwurf des agentiellen Realismus legt die Quantenphysikerin und feministische Wissenschaftstheoretikerin Karen Barad ein Konzept vor, welches Materie mit Diskurs verschränkt und daher an die humanismuskritischen und anthropozentrismuskritischen Arbeiten von Foucault anknüpft, aber in ihrer posthumanistischen Perspektive versucht, keine Trennung zwischen Natur und Kultur/Gesellschaft bzw. zwischen Materie und Diskurs einzuschreiben (Barad 2013, 52).

«Der Posthumanismus setzt nicht die Getrenntheit irgendeines (Dings) voraus, geschweige denn die vermeintliche räumliche, ontologische und erkenntnistheoretische Auszeichnung, die den Menschen absondert» (Barad 2012, 14). 
Hierbei geht es nicht darum, eine Grenze zwischen Menschen und Nicht-Menschen zu verwischen und sämtliche Unterschiede zu nivellieren, um damit den Humanismus einfach reziprok zu machen, sondern es geht um materialisierende Effekte der Grenzziehungen zwischen Menschen und Nicht-Menschen, die beide erst hervorbringen (Barad 2015b, 128).

Indem durch die digitalen Artefakte «die Welt» in einen digitalen Text verwandelt wird und damit in Gefahr gerät, zur reinen Kulisse zu mutieren (Meyer-Drawe 2018), wird das den Text generierende Medium in seiner materiellen wie diskursiven Verfasstheit unsichtbar gemacht (Latour 2015, 69). Mit diesem Mechanismus wird dieser nichtbesetzte Raum durch eine neuerliche Inthronisierung des Menschen besetzt. Dabei wird davon ausgegangen, dass das Medium - in seiner noch zu skizzierenden komplexen Verfasstheit - in der Verfügungsgewalt der menschlichen Akteure liegt (Dräger und Müller-Eiselt 2019). Obige Erwähnung der Infragestellung des Menschen durch das Digitale ist nur ein scheinbarer paradoxer Widerspruch zur Inthronisierung, denn beides ist eingebettet in das, was Bruno Latour die Verfassung der Moderne nennt. Einem zweifachen Akt, in dem zum ersten hybride Wesen geschaffen werden, die eine Mischung zwischen Natur und Kultur darstellen, und in dem zum zweiten eine Reinigungsarbeit betrieben wird, durch die zwei ontologisch vollkommen getrennte Zonen zwischen menschlichen und nichtmenschlichen Akteuren geschaffen werden (Latour 2015, 19).

«Die Moderne wird oft über den Humanismus definiert, sei es, um damit die Geburt des ‘Menschen` zu begrüßen, sei es um seinen Tod anzukündigen. Diese Gewohnheit jedoch ist selbst modern, denn sie bleibt asymmetrisch. Sie vergisst die gleichzeitige Geburt der «Nicht-Menschheit); die der Dinge oder Objekte ...» (Latour 2015, 22).

Diese skizzierte Asymmetrie ${ }^{1}$ zwischen Natur und Kultur inskribiert sich in die oben beschriebene Diskurstheorie, was aus der Perspektive eines material turn innerhalb der Geistes-, Kultur- und Sozialwissenschaften (Wunder 2020a) zu erneuten anthropozentrischen Umlaufbahnen führt (Barad 2012, 11). Es bleibt bei einer grundlegenden Trennung zwischen Ontologie und Epistemologie - mit einem Primat der Sprache (Latour 2016, 70).

«Der Sprache wurde zu viel Macht eingeräumt. Die sprachkritische Wende, die semiotische Wende. Es scheint, daß in jüngster Zeit bei jeder Wende jedes «Ding - selbst die Materialität - zu einer sprachlichen Angelegenheit oder einer anderen Form von kultureller Repräsentation wird. [...] Es geht um die Sprache. Es geht um den Diskurs. Es geht um die Kultur. In einer wichtigen Hinsicht ist das einzige, worum es anscheinend nicht mehr geht, die Materie» (Barad 2012, 7).

1 Zur Symmetrierung der Anthropologie im Kontext der Bildungsmedienforschung siehe Wunder 2018b. 
Selbst in Diskurstheorien, die die Materie mit einbeziehen, wie dies in Judith Butlers Werk Körper von Gewicht (Butler 1997) der Fall ist, bilden die Materie, ${ }^{2}$ die dort nur in Form von menschlichen Körpern in Erscheinung tritt, und der Diskurs eine Beziehung der «absoluten Äußerlichkeit» (ebd., 39). Mit der Verschränkung von Diskurs und Materie im agentiellen Realismus könnte die oben problematisierte Leerstelle theoretisch erfasst werden, um einen Zugang zu Orientierungswissen über (digitale) Medien in ihrer materiellen Verfasstheit, innerhalb der medienpädagogischen Forschung gewonnen werden.

\section{Agentieller Realismus als Onto-Epistemologie ohne anthropozentrische Erkenntnisakte}

Um die Verschränkung von Diskurs und Materie bzw. von Sozialkonstruktivsmus und Realismus ohne Widerspruch denkbar zu machen (Barad 2015a), entwickelt Barad den Begriff der Onto-Epistemologie, der anzeigt, dass beide sich nicht trennen lassen. Aus ihrer quantenphysikalischen Perspektive, speziell aus ihrer diffraktorischen Lesart (Barad 2013) der erkenntnistheoretischen Schriften von Niels Bohr (1964; 1966) kritisiert sie Ontologien, die auf der klassischen newtonschen Physik basieren. Hier kursiere die Annahme, dass es individuell abgrenzbare Objekte gebe, die spezifische Eigenschaften haben und auf einer Bühne - bestehend aus Raum und Zeit - als Akteurinnen und Akteure auftreten (Barad 2015c, 102). Vielmehr scheinen stattdessen Beschreibungen korrekt zu sein, welche von einem prozesshaften Werden eines Netzes ausgehen, bei dem Verbundenheit/eine Beziehungsstruktur, gedacht als ein Dazwischen, ohne einen materiellen Träger das Entscheidende ist (Dürr 2006,28 ). Wirklichkeit konstituiert sich also aus primitiven Relationen ohne präexistierende Relata (Barad 2012, 19). Eine solche ontologische Konzeption bleibt nicht ohne Folgen für die die Epistemologie, für die sich nunmehr kein Ort ausserhalb der Wirklichkeit bestimmen lässt, auch nicht in einem cartesianischen Subjekt, dem ein Blick von Nirgendwo (Nagel 2012) möglich ist (Barad 2012, 17). Barads Situierung der Epistemologie wird damit auf einen realistischen Grund gelegt - im Gegensatz zu Foucaults poststrukturalistisch-sozialkonstruktivistischem Epistembegriff, der zwar (erkennenden) Subjekten einen Platz in der Welt zuweist, diese aber nicht mehr in das fortlaufende Werden der Welt auf ganz fundamentale Art einbindet (ebd., 99). Der von Barad entwickelte Begriff der Intraaktion, der anstelle des Begriffs der Interaktion gesetzt wird, illustriert anschaulich die Untrennbarkeit von Epistemologie und Ontologie. Die Intraaktion setzt nämlich keine zuvor getrennten Entitäten voraus, die miteinander in kausaler Wechselwirkung stehen, wie dies der Begriff der

2 «Die Materie der Körper wird neu gefaßt als die Wirkung einer Machtdynamik, so daß die Materie der Körper nicht zu trennen sein wird von den regulierenden Normen, die ihre Materialisierung beherrschen, und von der Signifikation dieser materiellen Wirkungen» (Butler 1997, 22). 
Interaktion zu plausibilisieren sucht, sondern geht davon aus, dass Phänomene ${ }^{3}$ durch agentielle Schnitte in einer an und für sich unauftrennbaren Wirklichkeit geschaffen werden. Es wird quasi ein Aussen im Innen erzeugt. Demnach besteht die Wirklichkeit nicht aus Dingen an sich oder Dingen hinter den Phänomenen, sondern aus «Dingen-in-den-Phänomenen» (ebd., 21). An der Produktion von Phänomenen durch besagte agentielle Schnitte sind massgeblich Apparate beteiligt. Eine posthumanistische Auffassung des Apparats geht davon aus, dass diese materiell-diskursive Praktiken darstellen, d.h., die Apparate sind automatisch mit verschiedenen Begriffen und Konzepten verbunden, die automatisch andere Begriffe und Konzepte ausschliessen. Damit stellen Begriffe tatsächlich materiell-physikalische Anordnungen dar, die in Intraaktionen in einem breiten Netz ohne Grenzen eingebunden sind und dieses zugleich neu anordnen. In einer solchen Perspektive re-konfigurieren etwa Karten als diskursiv-materielle Apparate in Schulbüchern die Welt (Wunder 2020b).

Wir haben es damit mit einer «Materialität der Bedeutungserzeugung» (Barad 2012, 33) zu tun, welche den Dualismus zwischen Wort und Welt, Beobachtenden und zu Beobachtendem, Subjekt und Objekt negiert. Entscheidend ist, dass Apparate damit als Diskurspraktiken aufgefasst werden und nicht nur als Dinge, in denen sich Diskurspraktiken verkörpern (ebd., 35). Sie stellen Unterschiede her, die von Relevanz sind. Materie und Bedeutung, sprich Phänomene, deren Teil die Apparate sind, werden produziert. Die Welt wird durch sie (re-)konfiguiert (ebd., 31). Apparate sind die materiellen Bedingungen der Möglichkeit und Unmöglichkeit der Materialisierung und Relevanzbildung; sie setzen das in Kraft, was relevant ist und was vom Relevantsein ausgeschlossen ist. Apparate vollziehen agentielle Schnitte, die eindeutige Grenzen und Eigenschaften von «Entitäten` innerhalb von Phänomenen hervorbringen (ebd., 34-35).

Diskurs und Materie können demnach nicht getrennt werden. Die Welt artikuliert sich ständig in einer wechselseitigen Verschränkung von Materie und Diskurs, in dem keines dem anderen vorgeordnet ist. Vielmehr artikulieren sich beide wechselseitig (ebd., 32-43). «Die materiellen und semiotischen Apparate bilden ein nicht-dualistisches Ganzes» (Barad 2015a, 29). Materie ist ein tätiger Akteur, der am ständigen Werden der Welt beteiligt ist, sie ist damit diskurshaft (Barad 2012, 41). In Bezug auf die Erkenntnispraktiken, die in klassischen humanistischen Konzepten einen geistigen Agenten notwendig machen, um Verstehbarkeit zu realisieren, wird hier davon ausgegangen, dass Verstehbarkeit eine Leistung der Welt ist, deren einer Teil sich einem anderen Teil zu erkennen gibt, ohne hierbei schon eine erkennende Instanz präexistierend vorauszusetzen, sei diese menschlich oder nicht-menschlich.

3 Bei Niels Bohr weist der Begriff auf die Unzertrennlichkeit von zu messenden quantenphysikalischen Zuständen, Messapparat und Beobachtenden hin (Bohr 1966, 4). Barad nutzt den Begriff ganz ähnlich, erweitert ihn aber, indem er bei ihr auf die «ontologische Unzertrennlichkeit/Verschränkung intraagierender ‘Agentien〉 (agencies)» (Barad 2012, 19) hinweist. Damit wird sichergestellt, dass in Beschreibungen stets gewährleistet ist, dass Ganzheiten beschrieben werden und keine isolierten Gebilde (Barad 2015a, 26). 
«Menschliche Körper und menschliche Subjekte existieren als solche nicht schon zuvor; sie sind auch keine blossen Endprodukte. Menschen sind weder reine Ursachen noch reine Wirkungen, sondern ein Teil der Welt in ihrem unabgeschlossenen Werden.» (ebd., 37f.)

\section{Materialität als Orientierungsinstrument der Medienpädagogik in einer digitalen Welt}

(a) Erweiterter Blick. Der agentielle Realismus legt nahe, (digitale) Medien in ihrer materiell-semiotischen Verfasstheit als oben beschriebene Apparate aufzufassen, welche agentielle Schnitte in einer an und für sich unauftrennbaren Wirklichkeit vornehmen und damit ein Aussen im Innen erzeugen. Die Welt wird durch einen solchen Vorgang (re-)konfiguriert. Zu diesem Re-Konfigurationsvorgang gehört auch, dass entsprechende Positionen von Menschen und Nicht-Menschen zugewiesen werden, die in dieser Art vorher nicht existierend waren. Der erweiterte Blick für die Medienpädagogik ergibt sich dadurch, dass keine Entität - weder Mensch, Diskurs noch nicht-menschliche Akteure - in der Analyse priorisiert wird. Vielmehr kann im Rahmen einer flachen Onto-Epistemologie das gesamte Feld der Intraaktion in ihren Verschränkungen nachgezeichnet werden. Damit gewinnt man, so das Postulat, einen umfassenderen Blick vom Werden der Welt:

«Ich sage nicht nur, daß die vorhandenen Karten unvollständig sind, sondern daß sie Territorien mit einer derart unterschiedlichen Gestalt bezeichnen, daß sie sich mit unseren nicht einmal überlappen!» (Latour 2010, 286).

(b) Einbezug der Diskurstheorie. Die im ersten Teil entfalteten Überlegungen sind mit den Darlegungen zum agentiellen Realismus nicht hinfällig, erfahren aber dadurch eine Erweiterung und beugen der Tendenz vor, Diskurspraktiken als anthropomorphe Platzhalter in die Theoriearchitektur und den Forschungsprozess zu inskribieren (Barad 2012, 37). Übernommen werden kann zudem aus der Diskurstheorie ihr machtkritischer Ansatz (Butler 2015), in den nunmehr die Materie als eigenständiger Akteur mit eingebunden wird (Barad 2015d, 202). Grenzen sind keineswegs fest und naturmässig vorgegeben oder aktualisieren sich aus einer Sphäre jenseits von Raum und Zeit, sodass man Verantwortlichkeit für diese leugnen kann. Vielmehr sind sie interessengeleitete Instanzen von Macht, welche Relevanzsetzungen vornehmen und damit Ausschlüsse erzeugen (Barad 2015a, 48). Damit werden gezogene Grenzen, mit denen sich die medienpädagogische Forschung befasst - man denke an die Arbeiten zur digitalen Spaltung (Zillien und Haufs-Brusberg 2014) -, nicht allein in einer Wissensordnung oder sozioökonomischen Sphäre situiert, sondern die Macht wird innerhalb der Architektur der (digitalen) Apparate selbst verortet. So nehmen digitale Artefakte 
etwa Grenzziehungen zwischen vermeintlich fortschrittlichen Bildungssystemen vor, die durch das Operieren mit entsprechenden digitalen Endgeräten als solche markiert wurden, und rückständigen Bildungsregimen, die tendenziell ihre Lehre analog organisieren (Wunder 2018a, 141-151). Durch die Verschiebung von Grenzziehungen lassen sich Machtfragen an die Oberfläche bringen, welche die Mächtigen gerne zu verschütten versuchen (Barad 2015a, 59). Diese Perspektive kann der Medienpädagogik helfen, entsprechende Machtkonstellationen und Machteffekte zu identifizieren (Wunder 2020b).

(c) Erweiterung der Forschungsinstrumente. Durch die Verschränkung von Diskurs und Materie kann die medienpädagogische Forschung zur Generierung von Orientierungswissen in einer digitalen Welt sich solcher Forschungsinstrumente bedienen, welche genau dieser Verschränkung gerecht werden. Der Verfasser denkt hier insbesondere an die Situationsanalyse nach Clarke (Clarke 2012), da diese in der Lage ist, Diskurse und nichtmenschliche Akteure in Form von SituationMaps zu kartografieren und analytisch aufeinander zu beziehen. Allerdings müsste hierbei die Situierung der Situationsanalyse im interaktionistischen Paradigma durch ein intraaktionistisches Paradigma des agentiellen Realismus ergänzt werden, was auf methodischer Ebene im Grunde eine Art Mind-Switch erforderlich macht, um die beschriebenen Situationen mit der Terminologie des agentiellen Realismus zu erfassen. Ein stärkerer Umbau ist allerdings in Bezug auf die diskurstheoretische Ausrichtung und die sich daraus ergebene Methodologie notwendig. Denn mit der starken Orientierung der Situationsanalyse nach Clarke an Kellers Wissenssoziologischer Diskurstheorie werden menschliche Akteure wieder stark gemacht und dadurch kommt die Analyse von Diskursen mitsamt dem an der Grounded Theory orientierten Kodierparadigma als Diskursanalyse light daher (Diaz-Bone 2012, 16ff.). Das Kodierparadigma muss demzufolge - will man der post-humanistischen Perspektive des agentiellen Realismus und der Diskurstheorie nach Foucault gerecht werden - zum einen in ein diskursanalytisches Kodierparadigma überführt werden und muss sich zum anderen daran orientieren, dass der Post-Strukturalismus eben immer noch ein Strukturalismus - mit entsprechenden sozio-epistemischen Tiefenstrukturen - ist, die sich nicht einfach lesen lassen, sondern rekonstruiert werden müssen (Wunder 2018a, 62-66).

(d) Einbezug der ökologischen Dimension. Schliesslich wird die Medienpädagogik bei der Suche nach Orientierungswissen in einer digitalen Welt durch den agentiellen Realismus auf die fundamentale ökologische Dimension verwiesen, an die menschliche wie nicht-menschliche Akteure in digitalen Bildungsarrangements rückgebunden sind (Wunder 2020a). Der Diskurs über Digitalisierung bzw. digitale Bildung hat einen inhärenten blinden Fleck (Sühlmann-Faul und Rammler 
2018), der den Blick auf Fragen der Nachhaltigkeit ausblendet. So stellen sich konkret Fragen nach Energieverbrauch und dem damit zusammenhängenden $\mathrm{CO}_{2}$-Austoss. «Die Frage, wie viel Energie wir maximal umsetzen dürfen, wird für uns und andere Kreaturen tatsächlich zu einer Überlebensfrage» (Dürr 2010, 149). So betrug der Datendurchsatz im Jahr 1992 weltweit 100 GB pro Tag, im Jahr 1997 wurde die gleiche Menge pro Stunde und im Jahr 2002 pro Sekunde transferiert. Berechnungen zufolge soll der Datendurchsatz im Jahr 2021 bei 106.000 GB pro Sekunde liegen. So hinterlässt gegenwärtig das gesamte Internet einen Fussabdruck von 300 Millionen Tonnen $\mathrm{CO}_{2}$. Der Energieverbrauch stieg in Deutschland vom Jahr 2010 mit ca. 1.000 GWh zum Jahr 2014 auf 2.500 GWh an (Sühlmann-Faul und Rammler 2018, 46). Daneben stecken in den digitalen Geräten wertvolle Bodenschätze wie Erze, Metalle, Seltene Erden, die zum einen endlich sind (Schmidt 2018) und die zum anderen in anderen als Müll in den peripheren Regionen der Welt wieder auftauchen und dort Umwelt und Bevölkerung vergiften (SühlmannFaul und Rammler 2018, 24-25).

Auch hier, sieht man von Punkt (a) ab, könnte man diese Einsichten auch ohne den agentiellen Realismus gewinnen. Allerdings legt dieser mit seiner Onto-Epistemologie eine andere Perspektive nahe, welche auf den Menschen als epistemische Bezugsgrösse, gleich wie es die Diskurstheorie evoziert, verzichtet.

\section{Schluss}

Die von Foucault offerierte Trias aus Arbeit, Leben und Sprache, die zur Infragestellung des Menschen als epistemische Bezugsgrösse führt und demnach eine Wissenschaft bzw. medienpädagogische Forschung notwendig macht, welche ohne ein humanes Gravitationszentrum sichere Erkenntnisse zu erlangen sucht, kann im 21. Jahrhundert durchaus noch durch zwei weitere Grössen ergänzt werden, nämlich durch das Digitale und die ökologische Sphäre. Diese beiden zwingen im Grunde die Diskurstheorie in einen material turn (Coole 2014, 29) hinein, der stärker die Materie und materiellen Artefakte in ihrer Handlungs- und Diskursmächtigkeit berücksichtigt und damit die Trennung zwischen Natur und Kultur/Gesellschaft fraglich werden lässt. Anhand der Diskurstheorie von Michel Foucault und des agentiellen Realismus von Karen Barad wurde hier gezeigt, dass die Medienpädagogik zur Gewinnung von Orientierungswissen sich zum einen an Diskursen und Materialität gleichsam orientieren kann, ohne hierbei andererseits in ihren Theorien, Methodologien und Methoden anthropozentrisch zu werden. 


\section{Literatur}

Angermüller, Johannes. 2004. «Michel Foucault - auf dem Weg zum soziologischen Klassiker?» Soziologische Revue 27, 385-394. https://doi.org/10.1524/srsr.2004.27.4.385.

Bachelard, Gaston. 1978. Die Philosophie des Nein: Versuche einer Philosophie des neuen wissenschaftlichen Geistes. Mit einem Essay von Joachim Kopper und einer Einleitung von Gerhard Schmidt und Manfred Tietz. Wiesbaden: Heymann.

Bachelard, Gaston. 1987. Die Bildung des wissenschaftlichen Geistes: Beitrag zu einer Psychoanalyse der objektiven Erkenntnis. Frankfurt am Main: Suhrkamp.

Bächle, Thomas Christian. 2016. Digitales Wissen, Daten und Überwachung zur Einführung. Hamburg: Junius.

Balzer, Nicole. 2004. «Von den Schwierigkeiten, nicht oppositional zu denken. Linien der Foucault-Rezeption in der deutschsprachigen Erziehungswissenschaft». In Michel Foucault: Pädagogische Lektüren, hrsg. v. Norbert Ricken, 15-35. Wiesbaden: VS. https://doi. org/10.1007/978-3-322-85159-8_2.

Barad, Karen. 2012. Agentieller Realismus: Über die Bedeutung materiell-diskursiver Praktiken. Berlin: Suhrkamp.

Barad, Karen. 2013. «Diffraktionen: Differenzen, Kontigenzen und Verschränkungen von Gewicht». In Geschlechter Interferenzen: Wissensformen - Subjektivierungsweisen - Materialisierungen. hrsg. v. Corinna Bath, H. Meissner und St. Trinkaus, 27-67. Berlin: Lit.

Barad, Karen. 2015a. «Dem Universum auf halbem Wege begegnen: Realismus und Sozialkonstruktivismus ohne Widerspruch». In Verschränkungen, Karen Barad, 7-69. Berlin: Merve.

Barad, Karen. 2015b. «Die queere Performativität der Natur». In Verschränkungen, Karen Barad, 115-171. Berlin: Merve.

Barad, Karen. 2015c. «Dis/Kontinuitäten, RaumZeit-Einfaltungen und kommende Gerechtigkeit. Quantenverschränkungen und hantologische Erbschaftsbeziehungen». In Verschränkungen, Karen Barad, 71-113. Berlin: Merve.

Barad, Karen. 2015d. «Verschränkungen und Politik: Karen Barad im Gespräch mit Jennifer Sophia Theodor». In Verschränkungen, Karen Barad, 173-213. Berlin: Merve.

Bohr, Niels. 1964. Atomphysik und menschliche Erkenntnis I: Aufsätze und Vorträge aus den Jahren 1933-1955. 2. Aufl. Braunschweig: Friedrich Vieweg \& Sohn.

Bohr, Niels. 1966. «Atomphysik und Philosophie - Kausalität und Komplementarität: Beitrag zu 〈Philosophy in the Mid-Century〉». In Atomphysik und menschliche Erkenntnis II: Aufsätze und Vorträge 1958-1962, hrsg. v. Niels Bohr. Braunschweig: Friedrich Vieweg.

Bostrom, Nick. 2018. Superintelligenz: Szenarien einer kommenden Revolution. 3. Aufl. Berlin: Suhrkamp.

Butler, Judith. 1997. Körper von Gewicht: Die diskursiven Grenzen des Geschlechts. Frankfurt am Main: Suhrkamp.

Butler, Judith. 2015. Psyche der Macht: Das Subjekt der Unterwerfung. 8. Aufl. Frankfurt am Main: Suhrkamp. 
Clarke, Adele E. 2012. Situationsanalyse: Grounded Theory nach dem Postmodern Turn. Wiesbaden: Springer VS.

Coole, Diana. 2014. «Der neue Materialismus und Politik der Materialisierung». In Macht des Materials - Politik der Materialität. hrsg. v. Susanne Witzgall und Kerstin Stakemeier, 29-46. Zürich: Diaphanes.

Dahlmanns, Claus. 2008. Die Geschichte des modernen Subjekts: Michel Foucault und Norbert Elias im Vergleich. Münster: Waxmann.

Dander, Valentin. 2017. «Medien - Diskurs - Kritik. Potenziale der Diskursforschung für die Medienpädagogik». MedienPädagogik 27 (Spannungsfelder \& blinde Flecken), 50-64. https:// doi.org/10.21240/mpaed/27/2017.01.15.X

Diaz-Bone, Rainer. 2004. «Diskursive Kulturproduktion» Sociologia Internationalis 42 (1): 3769.

Diaz-Bone, Rainer. 2010. Kulturwelt, Diskurs und Lebensstil: Eine diskurstheoretische Erweiterung der Bourdieuschen Distinktionstheorie. 2., erweiterte Aufl. Wiesbaden: Springer VS. https://doi.org/10.1007/978-3-531-91940-9.

Diaz-Bone, Rainer. 2012. «Situationsanalyse - Strauss meets Foucault: Review Essay: Adele Clarke (2012). Situationsanalyse. Grounded Theory nach dem Postmodern Turn». Forum Qualitative Sozialforschung 14 (1): Art. 11. https://doi.org/10.17169/fqs-14.1.1928.

Diaz-Bone, Rainer, und Jennifer Widmer. 2018. «Zum Stand der Diskursforschung». Zeitschrift für Diskursforschung (2): 146-161. https://doi.org/10.3262/ZFD1802146.

Dräger, Jörg, und Ralph Müller-Eiselt. 2019. Wir und die intelligenten Maschinen: Wie Algorithmen unser Leben bestimmen und wir sie für uns nutzen können. München: DVA.

Dürr, Hans-Peter. 2006. Auch die Wissenschaft spricht nur in Gleichnissen: Die neue Beziehung zwischen Religion und Naturwissenschaften. 3. Aufl. Freiburg im Breisgau: Herder.

Dürr, Hans-Peter. 2010. Warum es ums Ganze geht: Neues Denken für eine Welt im Umbruch. 3. Aufl. München: Oekom.

Fink-Eitel, Hinrich. 1997. Michel Foucault zur Einführung. 3., durchges. Aufl. Hamburg: Junius.

Foucault, Michel. 1974. Die Ordnung der Dinge: Eine Archäologie der Humanwissenschaften. Frankfurt am Main: Suhrkamp.

Foucault, Michel. 1981. Archäologie des Wissens. 16. Aufl. Frankfurt am Main: Suhrkamp.

Foucault, Michel. 2000. Die Ordnung des Diskurses: [Inauguralvorlesung am Collège de France, 2. Dezember 1970]. 7. Aufl. Frankfurt am Main: Fischer.

Foucault, Michel. 2001a. «Die Geburt einer Welt». In Schriften in vier Bänden - Dits et écrits: Band 1 1954-1969, hrsg. v. Michel Foucault und Daniel Defert unter Mitarbeit von Jacques Lagrange, 999-1003. Frankfurt am Main: Suhrkamp.

Foucault, Michel. 2001b. «Die Prosa der Welt». In Schriften in vier Bänden - Dits et écrits: Band 1 1954-1969, hrsg. v. Michel Foucault und Daniel Defert unter Mitarbeit von Jacques Lagrange, 622-644. Frankfurt am Main: Suhrkamp.

Foucault, Michel. 2001c. «Interview mit Michel Foucault». In Schriften in vier Bänden - Dits et écrits: Band 1 1954-1969, hrsg. v. Michel Foucault und Daniel Defert unter Mitarbeit von Jacques Lagrange, 831-845. Frankfurt am Main: Suhrkamp. 
Foucault, Michel. 2001d. «Wer sind Sie, Professor Foucault? Gespräch mit P. Caruso». In Schriften in vier Bänden - Dits et écrits: Band 1, 1954-1969, hrsg. v. Michel Foucault und Daniel Defert unter Mitarbeit von Jacques Lagrange, 770-793. Frankfurt am Main: Suhrkamp.

Foucault, Michel. 2002. Die Geburt der Klinik: eine Archäologie des ärztlichen Blicks. 6. Aufl. Frankfurt am Main: Fischer.

Frietsch, Ute. 2014. «Die Ordnung der Dinge». In Foucault-Handbuch: Leben, Werk, Wirkung, hrsg. v. Clemens Kammler, Rolf Parr und Ulrich Johannes Schneider, 38-50. Stuttgart: Metzler.

Gehring, Petra. 2015. «Wird er sich auflösen? Foucaults Anthropologiekritik - ein Retraktandum». In Fines Hominis? herausgegeben von Marc Rölli. Bielefeld: transcript.

Gnosa, Tanja. 2019. «MachtDaten. Strategien digitaler Verdatung aus Foucault'scher Perspektive». In Diskurs der Daten: Qualitative Zugänge zu einem quantitativen Phänomen, hrsg. v. Pamela Steen und Frank Liedtke, 57-76. Berlin, Boston: De Gruyter. https://doi. org/10.1515/9783110609103-005.

Heidegger, Martin. 2010. Über den Humanismus. 11. Aufl. Frankfurt am Main: Klostermann Vittorio.

Kittler, Friedrich A. 1993. «Es gibt keine Software». In Draculas Vermächtnis: Technische Schriften, herausgegeben. von Friedrich A. Kittler, 225-242. Leipzig: Reclam.

Landwehr, Achim und Stefanie Stockhorst. 2008. Einführung in die europäische Kulturgeschichte. Paderborn: Schöningh.

Latour, Bruno. 2010. Eine neue Soziologie für eine neue Gesellschaft: Einführung in die AkteurNetzwerk-Theorie. Berlin: Suhrkamp.

Latour, Bruno. 2015. Wir sind nie modern gewesen: Versuch einer symmetrischen Anthropologie. 5. Aufl. Frankfurt am Main: Suhrkamp.

Latour, Bruno. 2016. Cogitamus. Berlin: Suhrkamp.

Loh, Janina. 2018. Trans- und Posthumanismus zur Einführung. Hamburg: Junius.

Meyer-Drawe, Käthe. 2018. Die Welt als Kulisse: Übertreibungen in Richtung Wahrheit. Paderborn: Ferdinand Schöningh.

Milzner, Georg. 2016. Digitale Hysterie: Warum Computer unsere Kinder weder dumm noch krank machen. Weinheim: Beltz.

Nagel, Thomas. 2012. Der Blick von nirgendwo. Frankfurt am Main: Suhrkamp.

Nassehi, Armin. 2019. Muster: Theorie der digitalen Gesellschaft. München: C.H. Beck.

Nietzsche, Friedrich. 1994. «Die fröhliche Wissenschaft Vorrede, Fünftes Buch». In Also sprach Zarathustra und andere Schriften, hrsg. v. Friedrich Nietzsche. Werke in drei Bänden, Bd. 2, 7-92. Köln: Könemann Verlagsgesellschaft.

Peirce, Charles S. 1991. Vorlesungen über Pragmatismus. Hamburg: Felix Meiner Verlag.

Precht, Richard David. 2018. Jäger, Hirten, Kritiker: Eine Utopie für die digitale Gesellschaft. 2. Aufl. München: Goldmann. 
Ricken, Norbert. 2007. «Von der Kritik der Disziplinarmacht zum Problem der Subjektivation: Zur erziehungswissenschaftlichen Rezeption Michel Foucault». In Foucault in den Kulturwissenschaften: Eine Bestandsaufnahme, hrsg. v. Clemens Kammler und Rolf Parr, 157-176. Heidelberg: Synchron Wiss.-Verl.der Autoren.

Rorty, Richard. 1975. The linguistic turn: Recent essays in philosophical method. Phoenix ed. Chicago, Ill. [u.a.]: Univ. of Chicago Press.

Sarasin, Philipp. 2006. Michel Foucault zur Einführung. 2., überarb. Aufl. Hamburg: Junius.

Schädelbach, Herbert. 1989. «Das Gesicht im Sand: Foucault und der anthropologische Schlaf». In Zwischenbetrachtungen: Im Prozeß Der Aufklärung; Jürgen Habermas Zum 60. Geburtstag, hrsg. v. Axel Honneth, 231-261. Frankfurt am Main: Suhrkamp.

Schmidt, Elisabeth. 2018. «Umweltschutz: Energieintensive Herstellung: Smartphones 2040 die größten Klimakiller». zdf.de - Nachrichten, 31.05.2018. https://web.archive.org/ web/20200110110239/https://www.zdf.de/nachrichten/heute/smartphones-2040-groesste-klimakiller-100.html.

Schneider, Ulrich Johannes. 2003. «Wissensgeschichte, nicht Wissenschaftsgeschichte». In Michel Foucault: Zwischenbilanz einer Rezeption; Frankfurter Foucault-Konferenz 2001, herausgegeben. von Axel Honneth, 220-229. Frankfurt am Main: Suhrkamp.

Schneider, Ulrich Johannes. 2004. Michel Foucault. Darmstadt: Primus.

Spitzer, Manfred. 2015. Cyberkrank! Wie das digitalisierte Leben unsere Gesundheit ruiniert. München: Droemer.

Sühlmann-Faul, Felix, und Stephan Rammler. 2018. Der blinde Fleck der Digitalisierung: Wie sich Nachhaltigkeit und digitale Transformation in Einklang bringen lassen. München: Oekom.

Witte, Egbert. 2005. «Michel Foucault im aktuellen erziehungswissenschaftlichen Diskurs.» In Zeitschrift für Erziehungswissenschaft 8 (2): 326-331.

Wittgenstein, Ludwig. 2000. Tractatus logico-philosophicus. Frankfurt am Main: Suhrkamp.

Wunder, Maik. 2018a. Diskursive Praxis der Legitimierung und Delegitimierung von digitalen Bildungsmedien. Bad Heilbrunn: Verlag Julius Klinkhardt.

Wunder, Maik. 2018b. «Symmetrische Anthropologie als reflexive Schlüsselkategorie zur Implementierung von digitaler Bildung» In Zeitschrift für internationale Bildungsforschung und Entwicklungspädagogik 41 (3): 31-35. https://doi.org/ 10.31244/zep.2018.03.08.

Wunder, Maik. 2020a. «Das Mitspracherecht der Dinge - Material turn und digitale Bildung». Bildung und Erziehung (73), 1, S. 76-90 https://doi.org/10.13109/buer.2020.73.1.76.

Wunder, Maik. 2020. «Re-Konfiguration der Welt durch Wanderungskarten. Diskursiv-materielle Aspekte eines Phänomens». In Migration und Bildungsmedien. Migration and Educational Media, herausgegeben von Sylvia Schütze und Eva Matthes, 290-304. Beiträge zur historischen und systematischen Schulbuch- und Bildungsmedienforschung. Bad Heilbrunn: Verlag Julius Klinkhardt.

Zillien, Nicole, und Maren Haufs-Brusberg. 2014. Wissenskluft und Digital Divide. Baden-Baden: Nomos. 